\title{
Relaxation times of kinetically constrained spin models with glassy dynamics
}

\author{
Nicoletta Cancrini ${ }^{1}$, Fabio Martinelli ${ }^{2}$, Cyril Roberto $^{3}$, and Cristina Toninelli ${ }^{*}$. \\ ${ }^{1}$ Dip.Matematica Univ. l'Aquila, 1-67100 L'Aquila, ITALY and INFM unitá Roma La Sapienza, \\ ${ }^{2}$ Dip.Matematica, Univ. Roma Tre, Largo S.L.Murialdo 00146, Roma, ITALY, \\ ${ }^{3}$ L.A.M.A., Univ Marne-la-Vallée, 5 bd Descartes 67454 Marne-la-Vallée FRANCE, \\ 4 I.H.E.S., Les Bois Marie 35, route de Chartres F-91440, Bures-sur-Yvette, FRANCE
}

\begin{abstract}
We analyze the density and size dependence of the relaxation time $\tau$ for kinetically constrained spin systems. These have been proposed as models for strong or fragile glasses and for systems undergoing jamming transitions. For the one (FA1f) or two (FA2f) spin facilitated FredricksonAndersen model at any density $\rho<1$ and for the Knight model below the critical density at which the glass transition occurs, we show that the persistence and the spin-spin time auto-correlation functions decay exponentially. This excludes the stretched exponential relaxation which was derived by numerical simulations. For FA2f in $d \geq 2$, we also prove a super-Arrhenius scaling of the form $\exp (1 /(1-\rho)) \leq \tau \leq \exp \left(1 /(1-\rho)^{2}\right)$. For FA1f in $d=1,2$ we rigorously prove the power law scalings recently derived in [13] while in $d \geq 3$ we obtain upper and lower bounds consistent with findings therein. Our results are based on a novel multi-scale approach which allows to analyze $\tau$ in presence of kinetic constraints and to connect time-scales and dynamical heterogeneities. The techniques are flexible enough to allow a variety of constraints and can also be applied to conservative stochastic lattice gases in presence of kinetic constraints.
\end{abstract}

Despite a great deal of theoretical and experimental efforts, the main issues in understanding liquid-glass or jamming transitions remain unsolved. These phenomena occur in different systems: supercooled liquids, colloidal suspensions, vibrated granular materials 1, 2]. Characteristic features of a glassy behavior include dramatic slowing down of dynamics when a proper external parameter is tuned (e.g. temperature for liquids) and the occurrence of a complex, non exponential and spatially heterogeneous [3] relaxation process. Experiments show that, if an ideal glass transition occurs, it exhibits mixed first and second order features. Indeed, even if the divergence of the relaxation time signals a second order like transition, no static diverging correlation length is detected. Furthermore relaxation times for fragile liquids diverge in a super-Arrhenius way: $\tau \simeq \exp \left(A\left(T-T_{c}\right) / T\right)$ with $A(x) \uparrow \infty$ as $x \downarrow 0$, a signal of a cooperative relaxation on increasingly large scales as $T$ decreases.

A class of microscopic models that have been proposed in the attempt of understanding these phenomena are the so-called kinetically constrained models $(\mathrm{KCM})(4,5,6$, 7, $8,9,10,11,12,13,14,15,16,17,18,19,20,21,22$ and references therein). KCM are systems of particles on a discrete lattice with no static interactions beyond hard core and evolution is given by a Markovian stochastic dynamics. The elementary move is either a jump of a particle to a nearby empty site in the conservative (Kawasaki) case or the creation/destruction of a particle in the non conservative (Glauber) case. In both situations the associated rates, if positive, verify detailed balance with respect to a simple product measure. However, and this is the most interesting feature, a move at site $x$ can occur only if the configuration around $x$ satisfies certain constraints. The latter mimic the local constraints that may occur in the physical systems and which may cause the dynamical arrest. Indeed, for a proper choice of the constraints, KCM show glassy features including stretched exponential relaxation, superArrhenius slowing down and dynamical heterogeneities. Several works and a great deal of numerical simulations have recently been devoted to understand the mechanism inducing these glassy properties and to derive the typical time-scales as well as the asymptotic form of correlation functions $(4,5,6,7,8,19,10,11,12,13,14,15,16,17$, 18, 19, 20, 21, 22 ). Numerical simulations are however very delicate because of the rapid divergence of $\tau$ as the density is increased. Furthermore, finite size effects often have non trivial scalings. In this work we present a new general probabilistic technique through which we obtain rigorous results on the dependence of $\tau$ on the size of the lattice and on the particle density, $\rho$. As a by-product we also obtain meaningful bounds on the long-time behavior of time correlations and persistence function.

We first introduce the models and our results and then sketch the technique, referring to 23] for rigorous proofs. We focus on non conservative models, also known as kinetically constrained spin models (KCSM) or facilitated spin models. Conservative models will be analyzed elsewhere [23]. KCSM are defined as follows. Each site $x$ can be occupied, $\eta_{x}=1$, or empty, $\eta_{x}=0$, and it changes its current state with rate $\left[(1-\rho) \eta_{x}+\rho\left(1-\eta_{x}\right)\right] f_{x}(\eta)$. Here $f_{x}(\eta)$ does not depend on $\eta_{x}$ and it encodes the kinetic constraint. Thus detailed balance is satisfied w.r.t. the Bernoulli product measure, $\mu_{\rho}$, at density $\rho$. KCSM can be divided into two classes: (i) non-cooperative and (ii) cooperative. In a non-cooperative model it is possible to completely empty any configuration containing somewhere a finite seed of vacancies. In a non-cooperative one that is not the case. The class (ii) can be further divided into: (iia) models that are ergodic in the thermodynamic 
limit at any $\rho<1$; (iib) models that display an ergodicity breaking transition at a critical density $\rho_{c}<1$. For the latter, above $\rho_{c}$, there exists an infinite spanning cluster of particles mutually blocked by the constraints. Finally (iib) can be classified as discontinuous or continuous according to the character of the percolation transition of the blocked structure.

Among non cooperative models we consider the one spin facilitated Fredrickson-Andersen model [5] (FA1f), which recently received a renewed attention as a model for strong glasses [11]. A move is allowed only if at least one of the nearest neighbors is empty: $f_{x}(\eta)=1$ if $\sum_{y \text { n.n.x }}\left(1-\eta_{x}\right)>0, f_{x}(\eta)=0$ otherwise. In [1] a dynamical field theory was derived which gives $(q=1-\rho)$ : $\tau \propto 1 / q^{z}$ with $z=3$ for $d=1, z=2+\epsilon(d)$ with $\epsilon(2) \simeq 0.3$, $\epsilon(3) \simeq 0.1$ and $\epsilon(d \geq 4)=0$. A recent exact mapping into a diffusion limited aggregation model, its renormalization and a careful treatment of the symmetries involved [13] gives instead $d=2$ as the upper critical dimension and $\epsilon(d)=0$ in $d \geq 2$.

Our results for FA1f are the followings. If $\tau$ denotes the inverse of the spectral gap of the Liouvillian operator generating the stochastic dynamics, i.e. the worst relaxation time on all one time quantities, we get: $\tau \propto 1 / q^{3}$ in $d=1,1 / q^{2}<\tau \leq 1 /\left(q^{2} \log 1 / q\right)$ in $d=2$ and $1 / q^{1+2 / d}<\tau \leq 1 / q^{2}$ in $d=3$. These rigorous results lead to $\epsilon(2)=0$ and $\epsilon(3) \leq 0$, disproving the findings in 11] and confirming those in 13]. Moreover our method allows us to identify explicitly the slowest modes which dominate relaxation. Furthermore, it is easily adapted to any possible choice of non cooperative constraints, e.g. to models in which more than one spin is needed to empty the whole lattice. We can also treat models with asymmetric (e.g. East model [6]) or partially asymmetric constraints $(7])$ which have been proposed to model fragile glasses [10] and the strong/fragile crossover. In particular for East, we obtain $\log \tau \simeq(\log (1 / q))^{2}$ as in [8, 9]. Finally we can deal with the persistence function

$$
F(t)=\int d \mu_{\rho}(\eta(0)) P\left[\eta_{0}(s)=\eta_{0}(0) \forall s<t\right] .
$$

In great generality 23] we prove that whenever the global relaxation time $\tau$ is finite, $F(t)$ decays exponentially fast. In particular, for FA1f we obtain $\tau_{F} \leq q^{-1} \tau$ for small $q$, where $\tau_{F}$ is defined via $F\left(\tau_{F}\right)=e^{-1}$. Although these findings disprove any stretched exponential decay at large times, for FA1f in $d=1$ our bound $\tau_{F} \leq 1 / q^{4}$ does not preclude the stretched form [11, 12] $F(t) \simeq \exp \left(-\sqrt{q^{3} t}\right)$ when $q \downarrow 0$ and $q^{3} t \simeq O(1)$. This is indeed the regime where numerical simulations are performed [12].

Among cooperative models without transition (iia) we consider the two spin facilitated FA model (FA2f) on square and cubic lattices. Here the constraint requires that at least two of the surrounding sites are empty in order for the rate to be non zero. Originally [5] a dynamical transition at a finite density was predicted but later it was shown that it cannot occur $14,15,25]$. Still, FA2f is relevant for fragile glasses since both super-Arrhenius divergence for $\tau$ and stretched exponential relaxation have been detected $5,14,15,16,17,18,19]$. Indeed, both the spin-spin time autocorrelation and the persistence function are fitted with $\exp \left(-(t / \tau)^{\beta}\right)$ with $\beta$ decreasing as the density $\rho$ is increased [16, 17, 18]. As pointed out in [4], beyond the general recognition that FA2f behaves like fragile glasses, little is known about the scaling of $\tau$. Different fits have been proposed: Adam-Gibbs form [15], Vogel-Fulcher form [16] and $\exp (c / q)$ [19]. The latter, which corresponds to a super-Arrhenius form when $q$ is rewritten in terms of the temperature, is supported by the conjecture that relaxation occurs via the diffusion of critical droplets of size $1 / q$ over distances $\exp (c / q)$ [14].

Our results for FA2f in $d=2$ and $d=3$ are the followings. We prove that $\exp (1 / q) \leq \tau \leq \exp \left(1 / q^{2}\right)$ which establishes a super-Arrhenius scaling and excludes the Vogel Fulcher form [16]. Furthermore, since our results hold uniformly on the system size, we obtain that relaxation in infinite volume is purely exponential at any fixed $q$. Furthermore, as for FA1f, we get a strict bound on the crossover regime where a stretched form may occur if we let simultaneously $q \downarrow 0$ and $t \uparrow \infty$. These findings contradict the asymptotic stretched exponential relaxation in $16,17,18$. The fit with a stretching exponent $\beta<1$ should be due to the rapid divergence of $\tau$, which was also a fitting parameter.

Among (iib) models with continuous transition, we consider the two dimensional North East model (NE). For NE both the up and right neighbors should be empty in order for a move to be allowed and a continuous transition occurs at the critical density of directed percolation, $\rho_{d p}$ [20]. Finally, for (iib) with a discontinuous transition, we consider the recently proposed Knight model 22]. We refer to 22] for the precise definition of the constraints; we only recall that the transition occurs again at $\rho_{d p}$ and has the remarkable features of an ideal glass transition. Indeed a finite fraction of the system is frozen at $\rho_{d p}$ and the size $\xi$ of blocked structures diverges faster than any power law as $\rho \uparrow \rho_{d p}$. This leads to a super-Arrhenius divergence if $\tau \simeq \xi^{z}$ is assumed.

Our main results for NE and Knights are the followings. For $\rho<\rho_{d p}$ we identify a constant which bounds $\tau$ uniformly on the system size, $L$. Therefore, even if an ergodicity breaking transition occurs, relaxation is purely exponential in the ergodic region. Moreover, we get that $\tau$ diverges when $\rho \nearrow \rho_{d p}$ and $\tau \propto \exp (L c(\rho))$ for $\rho>\rho_{d p}$. This result provides a possible test on the validity of Knights for systems undergoing jamming transitions.

Let us now sketch the main ideas beyond our approach, rigorous proofs will be reported elsewhere [23]. Here we focus on $d=2$. Results in $d=1$ can be derived analogously; we will comment at the end on $d=3$. We first introduce a new model referred to as the General Model $(G M)$ and derive results for its relaxation time, $\tau_{G M}$. 


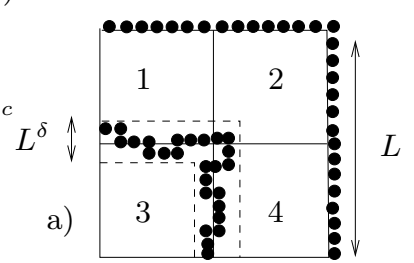

b)

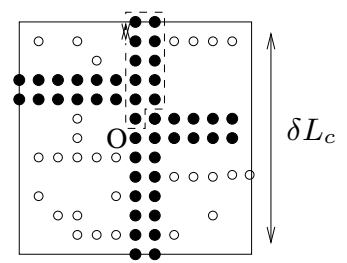

FIG. 1: a) Block dynamics for GM: percolating path of good sites $(\bullet)$ required to renew configuration on 3 . b) Blocking event for FA2f. - (o) stand for particles which do (do not) belong to the backbone. Sites inside dotted line form one of the sequences of $\geq \delta L_{c} / 2$ sites to be emptied before $O$.

Then we show how to map a given KCSM into GM by a renormalization technique. GM can be described as follows. Instead of two-valued occupation variables consider N-valued variables, $n_{x} \in S=(0, \ldots N-1)$, and a probability measure $\nu$ on $S$. We identify a subset $G$ of $S$ which we call the good event and we declare good a site $x$ if $n_{x} \in G$. The dynamics is defined as follows. Each site $x$ waits a mean one exponential time and then $n_{x}$ is refreshed by a new value $n_{x}^{\prime}$ sampled from $\nu$, provided its three North, North-East and East neighbors (i.e. $x+\vec{e}_{1}, x+\vec{e}_{1}+\vec{e}_{2}, x+\vec{e}_{2}, \vec{e}_{i}$ being the unit basis vectors) are good. If this constraint is not satisfied, $n_{x}$ remains unchanged. We consider GM on a square lattice $\Lambda_{L}$ of linear size $L$ with good boundary conditions on the top and right boundaries to ensure ergodicity. In order to evaluate $\tau_{G M}(L)$ we follow the bisection method 24]. Partition $\Lambda_{L}$ into four blocks as in fig (1) and define the following auxiliary accelerated dynamics. Each block waits a mean one exponential time and then its configuration is replaced by a new one chosen according to the product equilibrium probability given by $\nu$. On the top right block (block 2 in fig पa) ) this move is always allowed because of the boundary conditions. For the others, a constraint should be satisfied: on an l-shaped frame of width $L^{\delta}, \delta<1$, there should be a percolating cluster of good sites in the current configuration as in fig (1). In other words, the constraint requires the good GM boundary conditions on the blocks 1, 3, 4 (on 2 they are automatically guaranteed). The relaxation time $\tau_{G M}(L)$ is then bounded by the product

$$
\tau_{G M}(L) \leq \tau_{\text {block }}(L) \tau_{G M}(L / 2)
$$

with $\tau_{\text {block }}(L)$ the relaxation time for the block dynamics [28]. The above inequality corresponds intuitively to a two steps relaxation: first on the block scale, then inside each individual block. Notice that, trivially, $\tau_{G M}(L)$ is smaller than the worst case over the boundary conditions of the relaxation time on scale $L / 2$. However, such inequality is useless because without the good b.c. GM dynamics is not ergodic (i.e. $\tau$ is infinite). In other words, working with a constrained block dynamics is essential. Then, by dividing $\Lambda_{L / 2}$ into four blocks and so on up to constant size, we get

$$
\tau_{G M}(L) \leq c \prod_{n} \tau_{\text {block }}\left(2^{-n} L\right),
$$

where $c$ is a finite constant and the product contains $O(\log L)$ terms. Therefore we get a bound for $\tau_{G M}(L)$ which does not dependent on $L$ provided that the product over the $\tau_{\text {block }}$ 's converges. In turn this occurs if the probability that a site is good, $p$, is larger than a finite threshold, $p_{c}<1$. Indeed one can easily show that, for $p \simeq 1, \tau_{\text {block }}(L) \simeq\left(1+\exp \left(-m L^{\delta}\right)\right)$ where $\exp \left(-m L^{\delta}\right)$ comes from the probability that the constraint for the block dynamics on scale $\mathrm{L}$ is violated.

Let us now explain how to apply such a result to the KCSM models described above. We consider for definiteness FA2f in $d=2$ with empty boundary conditions on the top and right borders. We say that a region $V$ is internally spanned for the configuration $\eta$ if, when considering all the sites outside $V$ occupied, $\eta$ can be completely emptied using (internal) allowed moves. The probability for $\Lambda_{L}$ to be internally spanned has been evaluated in the context of bootstrap percolation: it goes to one exponentially fast when $L$ exceeds the crossover length $L_{c} \simeq \exp (c / q)$ with $c=\pi^{2} / 18$ [26]. Divide now the square lattice into disjoint blocks of size $k L_{c}, k \gg 1$, and identify each block by the coordinates of its center. On each of these renormalized sites we define a configuration space $S=\{0,1\}^{N_{b}}$, with $N_{b}$ the number of sites in a block. In $S$ we identify the good event $G$ as the set of configurations such that the block is internally spanned. Because of our choice of $k$ the probability that a renormalized site is good is $\simeq 1$. We can thus run the GM dynamics on the renormalized lattice and get $\sup _{L} \tau_{\mathrm{GM}}(L) \simeq 1$. Since in GM dynamics blocks are updated only if "enough" surrounding are internally spanned, by the two steps relaxation argument we get

$$
\tau(L) \leq \tau_{\mathrm{GM}}(L) \tau\left(k L_{c}\right) \simeq \tau\left(k L_{c}\right) .
$$

where $\tau(L)$ is the relaxation time for FA2f. In the infinite volume limit and for any $\rho<1$ this leads to an exponential relaxation for all one time functions as well as for the persistence function using a Feyman-Kac bound [23]. At the same time the density dependence of $\tau$ is completely encoded in the relaxation time on a lattice of size $L_{c}(\rho)$. To evaluate the latter we reduce the scale from $L_{c}$ to $1 / q^{2}$ via a strategy similar to the previous one. However, on scales smaller than $L_{c}$ the event "the block is internally spanned" becomes very unlikely and we are forced to make a different choice for the constraints of the renormalized dynamics in order to keep $\tau_{\mathrm{GM}} \simeq 1$. Our choice for the good event $G$ is suggested by the following two observations: (i) any empty segment of length $\ell$ can be displaced by one step in a given direction if there is at least one vacancy on the adjacent segment in that direction; (ii) the probability that there exists a fully occupied segment of length $\ell$ inside a critical square of size 
$L_{c}$ is very small as soon as $\ell \gg 1 / q^{2}$. Thus, we choose good events which force the not fully occupied condition on segments of length $1 / q^{2}$. By applying a bisection procedure analogous to the one used for GM together with elementary paths arguments, we get

$$
\tau\left(L_{c}\right) \leq c L_{c} \tau\left(1 / q^{2}\right)
$$

Finally we bound $\tau\left(1 / q^{2}\right)$ with the highest entropy cost to get $\tau_{F A}(L)<c L_{c} \exp \left(1 / q^{2}\right)=O\left(\exp \left(1 / q^{2}\right)\right)$.

The results for the other 2-dimensional models (FA1f, NE and Knight) are obtained analogously. Furthermore, for a generic choice of the constraints, we find that $\tau$ is dominated by $\tau\left(L_{c}\right)$ where $L_{c}$ is the crossover length for a region to be internally spanned (with the chosen constraints). Therefore $L_{c}$, which can be determined by a deterministic bootstrap-like procedure, is the relevant size over which numerical simulations should be performed. By analyzing the constraints one can also further reduce the problem to a much smaller scale, as we do for FA2f.

Lower bounds for $\tau$, directly in the thermodynamic limit, can be established either via a suitable choice of test functions in the variational characterization of $\tau$ or using our general result for the persistence function: $F(t) \leq \exp (-t q / \tau)$ [23]. Consider an event $B$, called the blocking event, and let $P_{B}(t)$ be the probability that the origin is occupied for all times $s \leq t$, worst case among all the starting configuration in $B$. The inequality for $F(t)$ implies $\mu_{\rho}(B) P_{B}(t) \leq \exp (-t q / \tau)$. We define the blocking event $B$ as the set of configurations for which, after standard bootstrap percolation (i.e. all possible FA2f moves in sequence) inside $\Lambda_{\delta L_{c}}$, a backbone of particles containing the origin survives. By choosing $\delta \ll 1$ we have $\mu_{\rho}(B) \simeq 1$. In infinite volume this backbone will eventually get unblocked thanks to the vacancies outside $\Lambda_{\delta L_{c}}$. However, this requires an ordered sequence of at least $\delta L_{c} / 2$ moves (fig \). Thus, $P_{B}\left(t=\epsilon \delta L_{c}\right) \simeq 1$ for sufficiently small $\epsilon$. Therefore $O(1) \leq \exp (-t q / \tau)$ for $t \simeq \epsilon \delta L_{c}$, i.e. $\tau \geq O\left(L_{c}\right)$. In $d=3$ lower bounds can also be obtained as before: using the results in [27] for $L_{c}$ we get $\tau \geq \exp (c / q)$ for FA2f and $\tau \geq \exp \exp (c / q)$ for FA3f. The latter is in agreement with the conjecture in 14 and disproves the possibility of an Adam Gibbs form [18] which corresponds to $\tau \simeq \exp (c / q)$. Finally the sharp lower bounds mentioned before for FA1f are obtained by a careful choice of a test function.

In summary, we have developed a technique which allows to obtain rigorous results on $\tau$ for KCSM via the knowledge of the typical regions which have to be rearranged to perform a movement. In other words, we have been able to connect rigorously time-scales and dynamical heterogeneities, a subject which have recently received great attention in the glass community [3]. The main new results established via this technique are super-Arrhenius behavior and exponential relaxation for cooperative models. Moreover our method suggests the typical length scale on which numerical simulations should be carried on. As for future developments, we believe that our technique can be applied to finite dimensional models other than KCM, e.g. models with static interactions beyond hard core and to conservative KCM models.

We thank G.Biroli for a careful reading of the manuscript and the IHES for its kind hospitality.

* cristina@corto.lpt.ens.fr

[1] P.G.De Benedetti and F.H.Stillinger Nature 410, 267 (2001); M.A.Ediger Ann.Rev.Phys.Chem. 51, 99 (2000)

[2] ER.Weeks et al. Science 287, 627 (2000); V.Trappe et al. Nature 411, (2001) 722; G.Marty, O.Dauchot Phys. Rev. Lett. 94, 015701 (2005)

[3] E.Vidal-Russel, N.E.Israeloff Nature 408, 695 (2000); L.A.Deschenes, D.A.Vande Bout Science 292255 (2001); L.Berthier et al. Science 3101797 (2005); C.Bennemann et al. Nature, 399, 246 (1999);O.Dauchot, G.Marty, G.Biroli, Phys.Rev.Lett. 95, 265701 (2005)

[4] Reviews: J. Jackle J. Phys. Cond. Matter 14, (2002) 1423; F. Ritort, P. Sollich Adv. in Phys. 52 (2003), 219.

[5] G.H.Fredrickson, H.C.Andersen Phys.Rev.Lett. 53, 1244 (1984); J.Chem.Phys. 84, 5822 (1985)

[6] J.Jackle, S.Eisinger Z.Phys.B: Cond.Mat. 84, 115 (1991)

[7] A.Buhot, J.P.Garrahan Phys.Rev. E 6421505 (2001)

[8] P.Sollich, M.R.Evans Phys.Rev.Lett 833238 (1999)

[9] D.Aldous, P.Diaconis J.Stat.Phys. 107845 (2002)

[10] J.P.Garrahan, D.Chandler Phys.Rev.Lett 89035704 (2002)

[11] S.Whitelam, L.Berthier, J.P.Garrahan, Phys. Rev.Lett 92, 185705 (2004); Phys.Rev.E 71, 026128 (2005)

[12] L.Berthier, J.P.Garrahan J.Chem.Phys. 119, 4367 (2003)

[13] R.Jack, P.Mayer, P.Sollich cond-mat/0601529

[14] J.Reiter J.Chem.Phys 95, 544 (1991)

[15] G.H.Fredrickson, S.A.Brawer J.Chem.Phys 84, 3351 (1986)

[16] I.S.Graham, L.Piché, M.Grant, J.Phys.Cond.Matt, 5, 6491 (1993); Phys.Rev.E, 55, 2132 (1997)

[17] P.Harrowell, Phys.Rev.E 48, 4359 (1993)

[18] G.H.Fredrickson Ann.Rev.Phys.Chem, 39, 149 (1988)

[19] S.Butler, P.Harrowell, J.Chem.Phys. 95, 4466 (1991)

[20] J.Reiter, F.Mauch, J.Jackle, Physica A, 184, 493 (1992); R.H.Schonmann, Ann. of Probab.,20 (1992), 174-193

[21] C.Toninelli, G.Biroli, D.S.Fisher Phys.Rev.Lett. 92185504 (2004)

[22] C.Toninelli,G.Biroli, D.S.FisherPhys.Rev.Lett 96035702 (2006) ; C.Toninelli, G.Biroli cond-mat/0512335

[23] N.Cancrini, F.Martinelli, C.Roberto, C.Toninelli in prep.

[24] F.Martinelli, Lectures Notes in Math., 1717, 93 (1999)

[25] M. Aizenmann, J.L. Lebowitz, J.Phys.A 21 (1988) 3801.

[26] A.E. Holroyd, Probab.Th.Rel.Fields 125 (2003) 195.

[27] R. Cerf, F. Manzo, Stoch.Proc.Appl. 101 (2002) 69.

[28] Here we are neglecting some unessential extra pre-factors arising from the overlapping of the $L^{\delta}$ boundary of a block with the other blocks, see [23] for a proper analysis. 